\title{
Sensing Properties of Thinned Phase-shifted Fiber Bragg Gratings
}

\author{
Jilei Yao, ${ }^{1}$ Shengli Pu, ${ }^{1,2 *}$ Yongxi Li, ${ }^{1}$ Rui Zhang, ${ }^{1}$ and Zixuan Jia ${ }^{1}$ \\ ${ }^{1}$ College of Science, University of Shanghai for Science and Technology, Shanghai 200093, China \\ ${ }^{2}$ Shanghai Key Laboratory of Modern Optical System, University of Shanghai for Science and Technology, \\ Shanghai 200093, China
}

(Received August 3, 2020; accepted September 10, 2020)

Keywords: phase-shifted fiber Bragg grating, side-polished fiber, refractive index, temperature, pump power

A $\pi$ phase-shifted fiber Bragg grating (PS-FBG) with reduced diameter is proposed for sensing applications. To achieve high sensitivity, etching and side polishing are used to decrease the diameter of the PS-FBG. The transmission peak undergoes a clear redshift with refractive index increase, and the sensitivity is $3815 \mathrm{pm} / \mathrm{RIU}$ when the surrounding refractive index ranges from 1.41514 to 1.45450 . The structure is also sensitive to ambient temperature from 25 to $55{ }^{\circ} \mathrm{C}$. Experimental results show that the temperature sensitivities are 12.26, 12.54, and $19.51 \mathrm{pm} /{ }^{\circ} \mathrm{C}$ for PS-FBGs with diameters of 125,87 , and $52 \mu \mathrm{m}$, respectively. The effect of the pump power on the sensing performance is also studied.

\section{Introduction}

A phase-shifted fiber Bragg grating (PS-FBG) is obtained by introducing a certain phase shift at a specific position of a normal Bragg grating, which changes the spectral distribution and forms a very narrow transmission peak in the transmission spectrum. ${ }^{(1,2)}$ PS-FBGs have been intensively investigated owing to their many desirable advantages, such as the very narrow transmission window and the flexible position of the resonance peak. ${ }^{(3)}$ PS-FBGs are widely used in the fields of distributed feedback lasers ${ }^{(4-6)}$ and narrowband filters. ${ }^{(7,8)}$ A PS-FBG has the characteristics of a Bragg grating, which can be used for high-precision sensing. ${ }^{(9-11)}$ The transmission peak undergoes a certain amount of shift with a change in the external environment, which is utilized to measure temperature, strain, and magnetic field. ${ }^{(12-16)}$ Because of its very narrow transmission peak, a PS-FBG has a high resolution. To improve the sensing performance, the diameter of a PS-FBG needs to be decreased to expose the evanescent field to the surrounding environment. ${ }^{(17,18)}$

Usually, two techniques (etching and side polishing) are employed to decrease the diameter of PS-FBGs to achieve high sensitivity. When using the etching technique, the mechanical strength of the as-fabricated grating will be relatively low. On the other hand, when using the side-polishing technique, it is difficult to control the polishing depth and the lateral surface is not uniform enough. In this work, a PS-FBG is side-polished before further etching. The ${ }^{*}$ Corresponding author: e-mail: shlpu@usst.edu.cn https://doi.org/10.18494/SAM.2020.3008 
combination of the two techniques can not only make the mechanical strength of PS-FBGs larger than that of PS-FBGs fabricated only by the etching technique, but also make the fiber core closer to the surrounding environment. Thus, the grating can be more sensitive to changes in the external environment.

\section{Experiment}

The length of the utilized $\pi$ PS-FBGs (provided by Puyang Institute of Opto-electrics Industrial Technology) is $15 \mathrm{~mm}$ and the center wavelength is $1550 \mathrm{~nm}$. The bandwidth is $0.3 \mathrm{~nm}$. Figure 1 shows a schematic of the laboratory-made side-polishing system. A PS-FBG is placed on the side-polishing platform; one end of the fiber is fixed and the other end is hung with a weight of around $15 \mathrm{~g}$ to keep the grating straight. The side-polishing time is $20 \mathrm{~min}$.

Figure 2(a) shows a typical microscopy image of a side-polished PS-FBG. The diameter of the narrowest part is about $87 \mu \mathrm{m}$. Then, the side-polished PS-FBG is fixed in a plastic U-shaped groove for hydrofluoric acid (HF 40\%) etching for 15 min to further reduce the diameter. To control the diameter of the grating precisely, it is further etched with lowconcentration HF (5\%) for another 5 min. A microscopy image of the side-polished FBG after twofold chemical etching is shown in Fig. 2(b). The thinnest part is only $52 \mu \mathrm{m}$. The thickness profiles of the side-polished and HF-etched fibers are shown in the bottom panels of Figs. 2(a) and 2(b), respectively. The profiles of the thinned gratings are roughly parabolic.

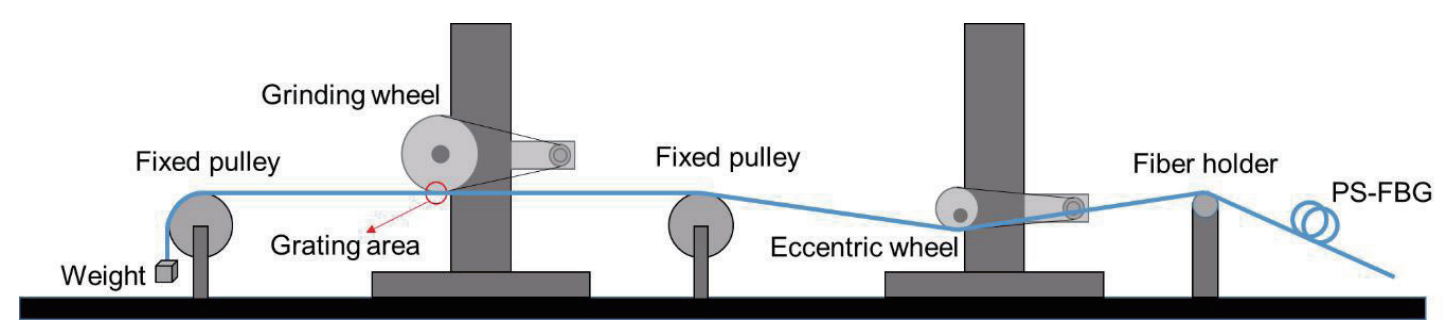

Fig. 1. (Color online) Schematic of the side-polishing system.

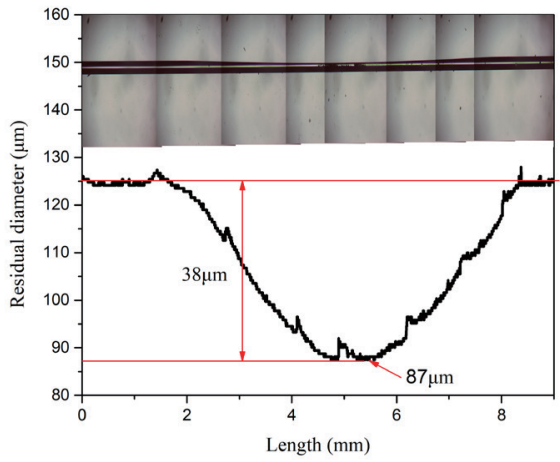

(a)

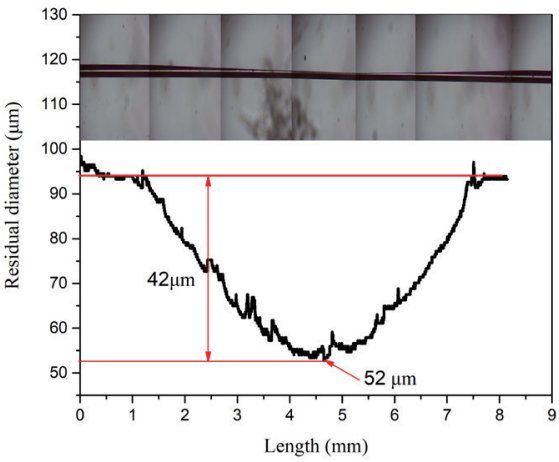

(b)

Fig. 2. (Color online) Microscopy images and profiles of side-polished and chemically etched PS-FBG with diameters of (a) 87 and (b) $52 \mu \mathrm{m}$. 
The experimental setup for investigating the sensing properties is shown in Fig. 3. Light is emitted from an amplified spontaneous emission (ASE) source and the transmitted light is detected by an optical spectrum analyzer (OSA). The ASE light source has a broadband range of $1500-1600 \mathrm{~nm}$ and the maximum output power is $10.2 \mathrm{~mW}$.

\section{Results and Discussion}

To investigate the effect of the surrounding refractive index on the PS-FBG spectrum, the structure is coated with glycerin solutions of different refractive indexes. The ambient temperature is controlled at about $20{ }^{\circ} \mathrm{C}$. After each measurement, the device is rinsed with water until the original spectrum in air is restored, indicating that no residual liquid is left. The transmission spectra are monitored in real time with an OSA with a resolution of $0.02 \mathrm{~nm}$. Figure 4 gives the transmission spectra of the device with the index increasing from 1.41514 to 1.45450 . The diameter of the PS-FBG is $52 \mu \mathrm{m}$. Figure 4 shows that the transmission peak shifts to a longer wavelength with increasing refractive index. When the external refractive index is larger than that of the fiber core (1.447), the transmission markedly decreases, as can be seen from the spectrum when the surrounding index is 1.45450. The relationship between the wavelength of the transmission peak and the refractive index is given in Fig. 5. An almost

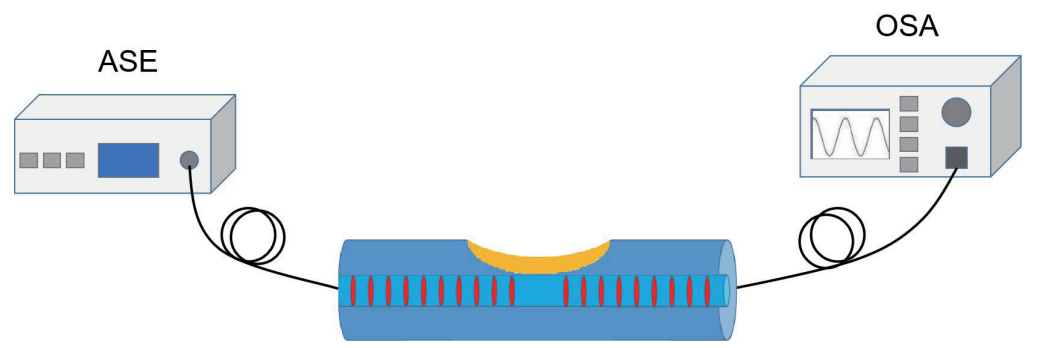

Fig. 3. (Color online) Experimental setup for investigating the sensing properties.

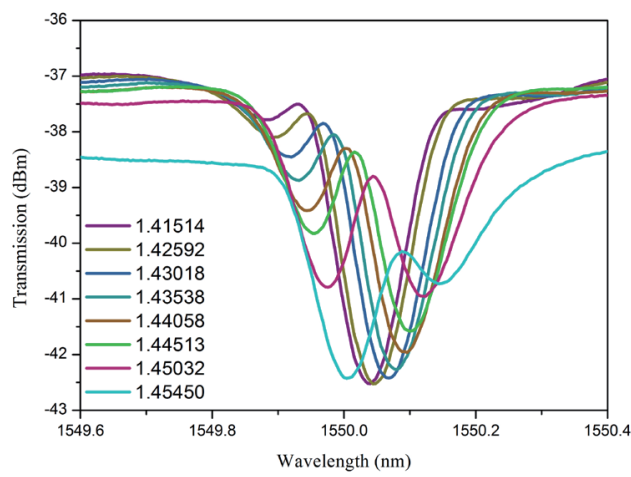

Fig. 4. (Color online) Transmission spectra of PS-FBG coated with solutions with refractive indexes in the range of 1.41514-1.45450. The diameter of the PS-FBG is $52 \mu \mathrm{m}$.

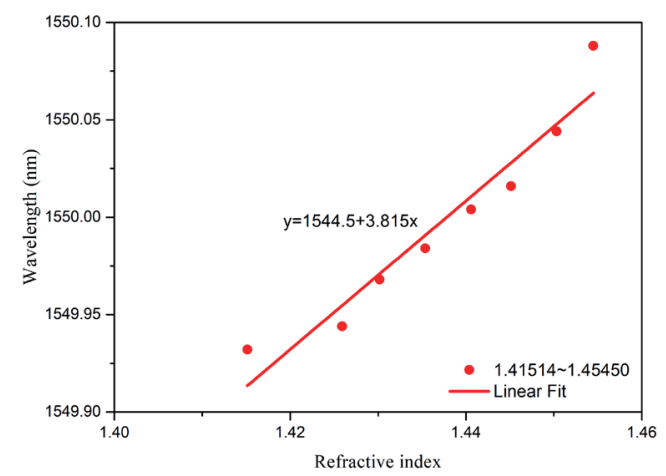

Fig. 5. (Color online) Peak wavelength of PS-FBG as a function of surrounding refractive index. The diameter of the PS-FBG is $52 \mu \mathrm{m}$. 
linear relationship is obtained and the sensitivity is $3815 \mathrm{pm} / \mathrm{RIU}$. The obtained sensitivities are much higher than that of a $\pi$ PS-FBG fabricated on an etched side-hole fiber $(-278.5 \mathrm{pm} / \mathrm{RIU}){ }^{(17)}$

To investigate the effect of temperature, PS-FBGs are placed into a temperature control chamber. The temperature is gradually increased from 25 to $55{ }^{\circ} \mathrm{C}$ with a step of $5{ }^{\circ} \mathrm{C}$. Figure 6 shows the transmission spectra of PF-FBGs with different diameters at different temperatures. As the temperature increases, the redshift of the peak wavelength is clearly observed. The relationship between the wavelength shift and temperature is given in Fig. 7; a linear relationship is found. The temperature sensitivities are $12.26,12.54$, and $19.51 \mathrm{pm} /{ }^{\circ} \mathrm{C}$ for thinned PS-FBGs with diameters of 125,87 , and $52 \mu \mathrm{m}$, respectively. The structure with a thinner fiber diameter has a larger sensitivity, which may be assigned to the larger evanescent field for the thinner grating. In addition, when the temperature is fixed, the peak shifts to a longer wavelength with decreasing diameter.

The resonance wavelength of an FBG is expressed as ${ }^{(16)}$

$$
\lambda=2 n_{\text {eff }} \Lambda,
$$

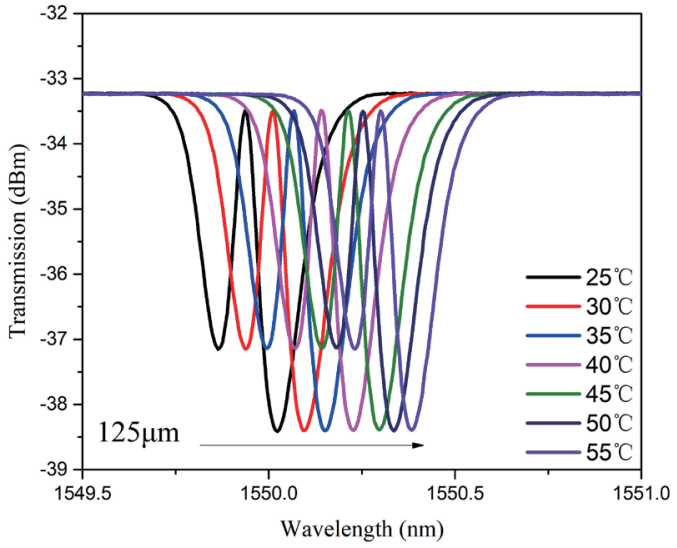

(a)

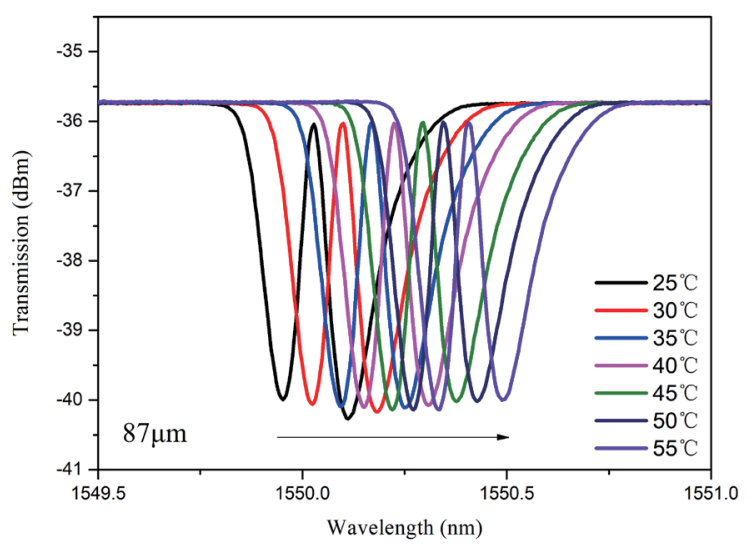

(b)

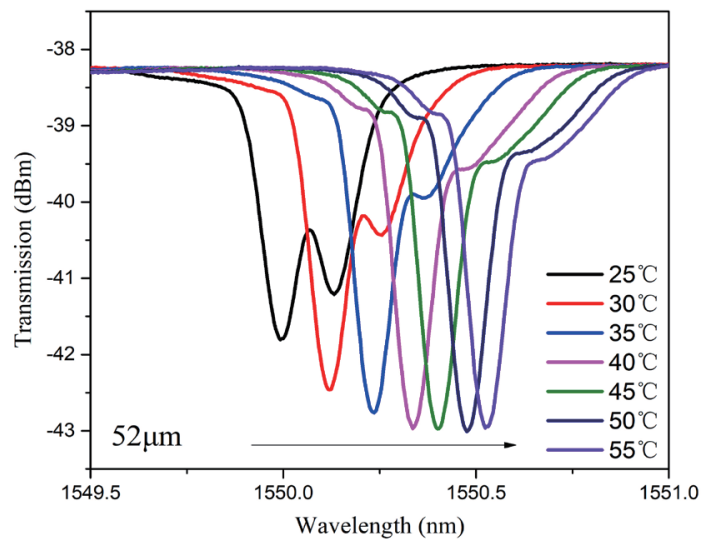

(c)

Fig. 6. (Color online) Transmission spectra at different temperatures for PS-FBGs with diameters of (a) 125, (b) 87 , and (c) $52 \mu \mathrm{m}$. 


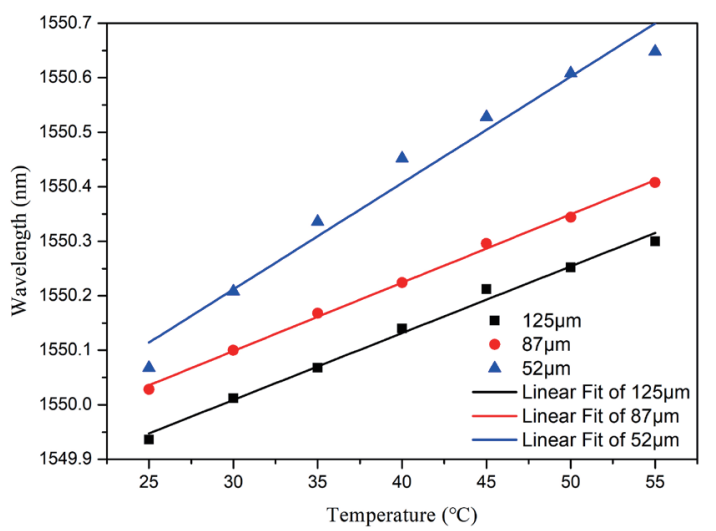

Fig. 7. (Color online) Wavelength shift as a function of temperature for PS-FBGs with different diameters.

where $\Lambda$ is the grating period and $n_{\text {eff }}$ is the effective index. Any change in either of these two parameters will cause the resonant wavelength to change. The refractive index and grating period at any temperature $T$ are given by

$$
\begin{aligned}
& n=n_{0}\left[1+\alpha\left(T-T_{0}\right)\right], \\
& n=\Lambda_{0}\left[1+\beta\left(T-T_{0}\right)\right],
\end{aligned}
$$

where $n_{0}$ and $\Lambda_{0}$ are the effective refractive index and grating period at temperature $T_{0}$, respectively.

Then, the resonant wavelength of a Bragg grating is expressed as

$$
\lambda^{\prime}=2 n_{0}\left[1+\alpha\left(T-T_{0}\right)\right] \cdot \Lambda_{0} \cdot\left[1+\beta\left(T-T_{0}\right)\right]
$$

The thermo-optical coefficient of a fiber is $\alpha \approx 1.1 \times 10^{-5} /{ }^{\circ} \mathrm{C}$ and the thermal expansion coefficient is $\beta \approx 5.2 \times 10^{-7} /{ }^{\circ} \mathrm{C}$. ${ }^{(19)}$ Because $\alpha \ll 1$ and $\beta \ll 1$, Eq. (4) can be simplified to

$$
\lambda^{\prime} \approx \lambda_{0}\left[1+\gamma\left(T-T_{0}\right)\right]
$$

where $\lambda_{0}=2 n_{0} \Lambda_{0}$ is the center wavelength of the Bragg grating when the temperature is $T_{0}$. $\gamma=\alpha+\beta$ is the temperature coefficient including the thermo-optic and thermal expansion effects. Therefore, the variation of the wavelength at temperature $T$ is written as

$$
\Delta \lambda=\lambda^{\prime}-\lambda_{0} \approx \lambda_{0} \gamma\left(T-T_{0}\right)
$$


Thus, the shift of the central wavelength of the PS-FBG transmission peak is linear with the temperature change, which is in good agreement with the experimental results.

To investigate the effect of the incident power, a coupler and a pump source are inserted into the experimental setup, which is schematically shown in Fig. 8. A $3 \mathrm{~dB}$ coupler is used to connect the broadband light source with a wavelength ranging from 1500 to $1600 \mathrm{~nm}$, a $980 \mathrm{~nm}$ pump source, and a PS-FBG. The incident power of the broadband light source is fixed at $10.2 \mathrm{~mW}$. Figure 9(a) shows the transmission spectra of a 52- $\mu \mathrm{m}$-diameter PS-FBG when the pump power increases from 0 to $100 \mathrm{~mW}$ with an interval of $10 \mathrm{~mW}$. The transmission spectrum redshifts with increasing incident pump power. The relationship between the wavelength shift and pump power is given in Fig. 9(b). The pump power responsivity is $4 \mathrm{pm} / \mathrm{mW}$, which is relatively low compared with the refractive index and temperature sensitivities. However, for high-precision measurement, it may be necessary to use a power-stabilized source.

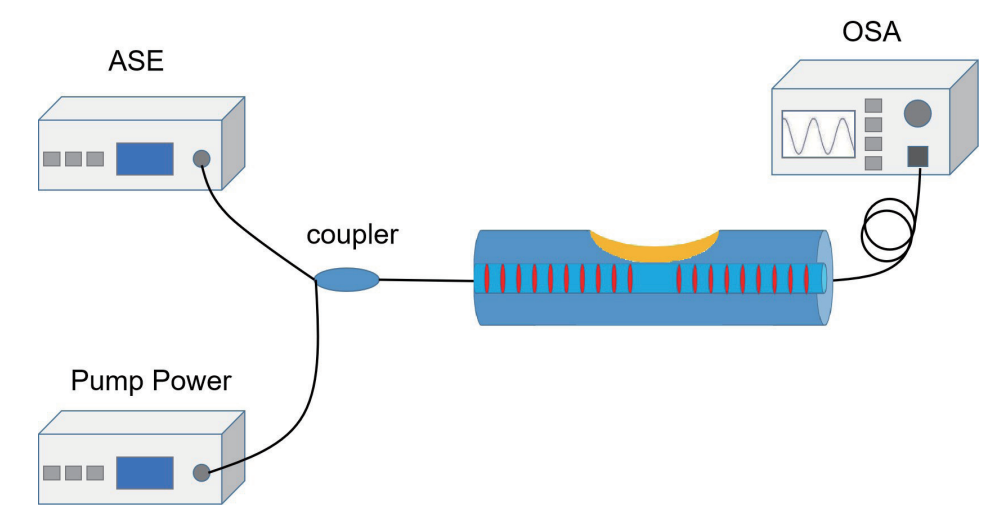

Fig. 8. (Color online) Experimental setup for investigating the effect of the incident pump power.

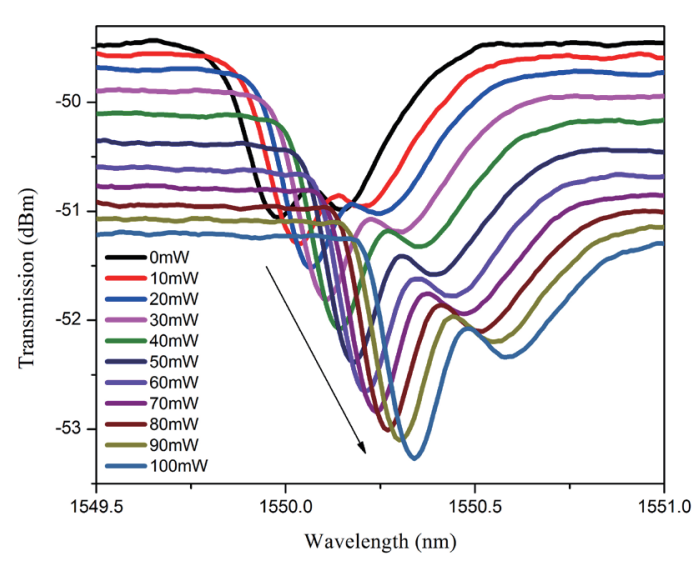

(a)

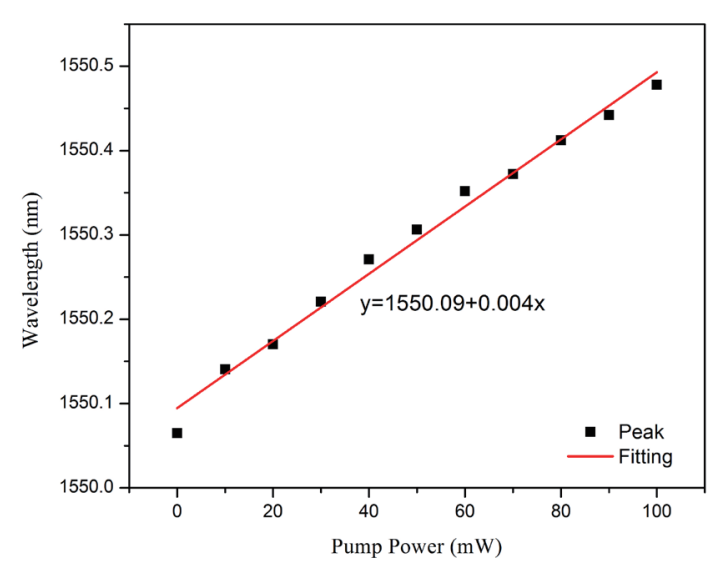

(b)

Fig. 9. (Color online) (a) Transmission spectra of PS-FBG with diameter of $52 \mu \mathrm{m}$ at different pump powers. (b) Wavelength shift as a function of pump power. 


\section{Conclusion}

The diameter of a $\pi$ PS-FBG was decreased using the side-polishing and etching techniques. When the residual diameter was $52 \mu \mathrm{m}$, the wavelength of the transmission peak clearly showed a redshift when the refractive index increased from 1.41514 to 1.44513 . When the refractive index was 1.45450 , the transmission markedly decreased. An almost linear relationship between the wavelength of the transmission peak and the refractive index was obtained and the sensitivity was $3815 \mathrm{pm} / \mathrm{RIU}$ when the refractive index was $1.41514-1.45450$. The transmission peak shifted to a longer wavelength with increasing temperature. The temperature sensitivities were $12.26,12.54$, and $19.51 \mathrm{pm} /{ }^{\circ} \mathrm{C}$ for PS-FBGs with diameters of 125,87 , and $52 \mu \mathrm{m}$, respectively. For the PS-FBG with a diameter of $52 \mu \mathrm{m}$, the pump power responsivity was $4 \mathrm{pm} / \mathrm{mW}$.

\section{Acknowledgments}

This research was supported by the National Natural Science Foundation of China (Grant No. 61675132), Shanghai "Shuguang" Program (Grant No. 16SG40), Shanghai Talent Development Fund (Grant No. 201529), and Key Laboratory of Specialty Fiber Optics and Optical Access Networks, Joint International Research Laboratory of Specialty Fiber Optics and Advanced Communication (No. SKLSFO2014-05).

\section{References}

1 X. F. Chen, J. P. Yao, F. Zeng, and Z. C. Deng: IEEE Photonics Technol. Lett. 17 (2005) 1390. https://doi. org/10.1109/Lpt.2005.848408

2 A. Melloni, M. Chinello, and M. Martinelli: IEEE Photonics Technol. Lett. 12 (2000) 42. https://doi. org/10.1109/68.817464

3 G. P. Agrawal and S. Radic: IEEE Photonics Technol. Lett. 6 (1994) 995. https://doi.org/10.1109/68.313074

4 P. Liu, W. Z. Huang, W. T. Zhang, and F. Li: Opt. Lett. 43 (2018) 2499. https://doi.org/10.1364/O1.43.002499

5 A. Tehranchi, S. Loranger, and R. Kashyap: IEEE J. Quantum Electron. 54 (2018) 1. https://doi.org/10.1109/ jqe.2018.2820218

6 S. Loranger and R. Kashyap: Opt. Lett. 43 (2018) 5705. https://doi.org/10.1364/O1.43.005705

7 J. Sun, Z. Wang, M. Wang, Z. Zhou, N. Tang, J. Chen, and X. Gu: Appl. Opt. 56 (2017) 9114. https://doi. org/10.1364/AO.56.009114

8 A. L. Zhang, L. Y. Hao, B. Geng, and D. Li: Opt. Laser Technol. 116 (2019) 1. https://doi.org/10.1016/ j.optlastec.2019.03.007

9 J. G. Chen, Q. W. Liu, and Z. Y. He: J. Lightwave Technol. 35 (2017) 4838. https://doi.org/10.1109/ Jlt.2017.2760343

10 C. A. F. Marques, A. Pospori, L. Pereira, S. Marques, P. Antunes, O. Bang, D. J. Webb, and P. Andre: 2017 SBMO/IEEE MTT-S International Microwave and Optoelectronics Conference (IMOC) 17396077. https://doi. org/10.1109/IMOC.2017.8121046

11 H. Z. Zhai, Q. Wu, K. Xiong, and R. Wang: IEEE Photonics Technol. Lett. 31 (2019) 1335. https://doi. org/10.1109/Lpt.2019.2926849

12 Y. J. Jiang, Y. Yuan, J. Xu, D. X. Yang, D. Li, M. R. Wang, and J. L. Zhao: Opt. Lasers Eng. 86 (2016) 236. https://doi.org/10.1016/j.optlaseng.2016.06.011

13 A. Halstuch and A. A. Ishaaya: Opt. Lett. 43 (2018) 3893. https://doi.org/10.1364/O1.43.003893

14 C. Hnatovsky, D. Grobnic, and S. J. Mihailov: Opt. Express 26 (2018) 23550. https://doi.org/10.1364/ Oe.26.023550

15 L. F. Bao, X. Y. Dong, S. Q. Zhang, C. Y. Shen, and P. P. Shum: IEEE Sens. J. 18 (2018) 4008. https://doi. org/10.1109/Jsen.2018.2820741 
16 J. J. Sun, Z. F. Wang, M. Wang, X. M. Xi, and J. B. Chen: Acta Opt. Sinica 37 (2017) 1006004. https://doi. org/10.3788/AOS201737.1006004

17 Q. Zhang, L. Hu, J. Tian, N. J. Ianno, and M. Han: Proc. SPIE 8722 (2013) 87220R. https://doi. org/10.1117/12.2018265

18 X. F. Huang, Z. M. Chen, L. Y. Shao, K. F. Cen, D. R. Sheng, J. Chen, and H. Zhou: Appl. Opt. 47 (2008) 504. https://doi.org/Doi 10.1364/Ao.47.000504

19 R. Kashyap: Fiber Bragg Gratings, R. Kashyap, Ed. (Academic Press, London, 2010) 2nd ed., Chap. 2. https:// doi.org/10.1016/B978-0-12-372579-0.00002-8

\section{About the Authors}

Jilei Yao received her B.S. degree in electronic information science and technology from Wenzheng College of Soochow University. She is currently pursuing her master's degree at the College of Science, University of Shanghai for Science and Technology. (1767975014@qq.com)

Shengli Pu received his Ph.D. degree in 2006 from Shanghai Jiao Tong University. He is currently a professor at the College of Science, University of Shanghai for Science and Technology. He was a visiting associate professor at Cornell University from August 2012 to August 2013. He has (co-)authored over 100 peer-reviewed papers in physics and optics. His research interests focus on advanced photonic materials and devices, especially the novel optical properties and photonic applications of magnetic fluids/ferrofluids. (shlpu@usst.edu.cn)

Yongxi Li received his B.S. degree from the College of Science, University of Shanghai for Science and Technology. He is currently pursuing his master's degree at the same univiersity. (984596702@qq.com)

Rui Zhang received his B.S. degree from the College of Physics and Photoelectric Engineering, Taiyuan University of Technology. He is currently pursuing his master's degree at the College of Science, University of Shanghai for Science and Technology. (zhangrui-2012@foxmail.com)

Zixuan Jia received his B.S. degree in physics from Ludong University. He is currently pursuing his master's degree at the College of Science, University of Shanghai for Science and Technology. (15552260662@163.com) 